\title{
Coupled Ladders in a Magnetic Field
}

\author{
T. Giamarchi \\ Laboratoire de Physique des Solides, CNRS URA 02, U.P.S. Bât 510, 91405 Orsay, France
}

\author{
A. M. Tsvelik \\ Department of Physics, University of Oxford, 1 Keble Road, Oxford, OX1 3NP, United Kingdom
}

(October 1, 2018)

\begin{abstract}
We investigate the phase transitions in two-legs ladder systems in the incommensurate phase, for which the gap is destroyed by a magnetic field $\left(h_{c 1}<h\right)$ and the ladder is not yet totally saturated $\left(h<h_{c 2}\right)$. We compute quantitatively the correlation functions as a function of the magnetic field for an isolated strong coupling ladder $J_{\perp} \gg J_{\|}$and use it to study the phase transition occuring in a three dimensional array of antiferromagnetically coupled ladders. The three dimensional ordering is in the universality class of Bose condensation of hard core bosons. We compute the critical temperature $T_{c}(h)$ as well as various physical quantities such as the NMR relaxations rate. $T_{c}$ has an unusual camel-like shape with a local minimum at $h=\left(h_{c 1}+h_{c 2}\right) / 2$ and behaves as $T_{c} \sim\left(h-h_{c 1}\right)^{2 / 3}$ for $h \sim h_{c 1}$. We discuss the experimental consequences for compounds such as $\mathrm{Cu}_{2}\left(\mathrm{C}_{5} \mathrm{H}_{12} \mathrm{~N}_{2}\right)_{2} \mathrm{Cl}_{4}$
\end{abstract}

\section{INTRODUCTION}

There has been recently considerable interest $t$ on spin ladder materials. These systems, quite remarkably, have a gap in the spin excitation spectrum for an even number of legs and no gap for an odd number. Thisphenomenon, reminiscent of the Haldane conjecture been explored in oreat details both theoretically $[11$ and experimentally 12 it

In ladders, contrarily to the case of spin $S$ chains, the gap and the dispersion in the ladder are controlled by two different energy scales, namely the transverse $J_{\perp}$ and longitudinal $J_{\|}$exchanges. The ladders are thus prime candidates to study quantum phase transitions where the spin gap is destroyed by application of a magnetic field. Because of this separation of energy scales between the gap and the exchange, even when the gap is destroyed quantum effects are still crucial. The ladders thus offers the possibility of an extremely rich quantum behavior, unsuspected in more conventional spin systems. Such quantum phase transitions were indeed studied experimentally $14.17,18$. On the theoretical side they were investigated using a bosonization technique 9 for a single ladder. Close to the critical point where the gap vanished, the spin-spin correlation functions were found to diverge with a universal exponent, leading to a divergent NMR relaxation rate $1 / T_{1} \sim T^{-1 / 2}$, in good agreement with the experimental findings. Between the critical field $h_{c 1}$ where the gap was destroyed and the saturation field $h_{c 2}$, the ladder had incommensurate spin spin correlation function with a quite distinctive spectrum compared to single chain systems. These results were confirmed and extended in subsequent analytical and numerical calculation 22023.

Due to the gaped nature of the excitations for a single ladder when $h<h_{c 1}$ a weak interladder coupling is inefficient and the single ladder approximation is nearly exact. This is clearly different in the incommensurate phase $h_{c 1}<h<h_{c 2}$, and the question of the coupling of ladders becomes much more crucial. Quite generally interladder coupling can lead now to a three dimensional ordered phase. This is the case for example for the compound $\mathrm{Cu}_{2}\left(\mathrm{C}_{5} \mathrm{H}_{12} \mathrm{~N}_{2}\right)_{2} \mathrm{Cl}_{4}$ which has an experimentally accessible gap of $\Delta \sim 11 K$. Specific heat measurements have revealed the existence of a transition at finite tem perature, the nature of which is still controversial 182426 . It is thus a challenge, both from a theoretical point of view and in view of application to experiments, to understand how three dimensional ordering can occur in ladder systems.

We investigate the nature and physical properties of such transition by looking at antiferromagnetically coupled ladders. Because of the peculiar nature of the excitation spectrum in ladders, this transition is different from the one occuring in more conventional spin materials.

The plan of the paper is as follows. In section II we define the model for coupled ladders. For simplicity we confine ourselves to the case of strongly coupled ladders $J_{\perp} \gg J_{\|}$. In section III we examine the single ladder in this limit, using a mapping on a single spin chain20, 28.21. We compute quantitatively the correlation functions as a function of the magnetic field. The weak and strong coupling limits give an identical structure for the correlations functions and we recover the universal exponents and spectrum for the spin-spin correlation functions derived in Ref. 19. The three dimensionally coupled ladders are described in section IV. The three dimensional ordering is in the universality class of Bose condensation. We compute the critical temperature $T_{c}$ as well as various physical quantities such as the NMR relaxations rate. $T_{c}$ has a camel-like shape with a local minimum at $h=\left(h_{c 1}+h_{c 2}\right)$ and behaves as $T_{c} \sim\left(h-h_{c 1}\right)^{2 / 3}$ for $h \sim h_{c 1}$. We discuss the experimental consequences for compounds such as $\mathrm{Cu}_{2}\left(\mathrm{C}_{5} \mathrm{H}_{12} \mathrm{~N}_{2}\right)_{2} \mathrm{Cl}_{4}$. Conclusions can 
be found in section $\mathrm{V}$ and some technical details are left for the Appendix.

\section{THE MODEL}

We consider the two legs ladders shown in Figure 1.

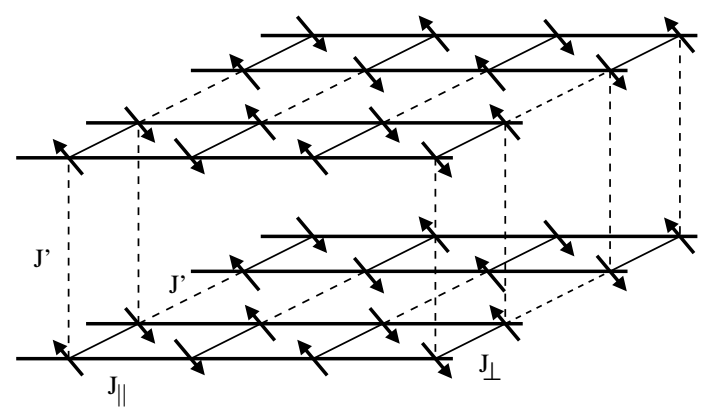

FIG. 1. The two leg ladder system considered in this paper. An interladder coupling $J^{\prime}$ couples the ladder in a three dimensional way.

For the moment we consider a single ladder and thus take $J^{\prime}=0$. The ladder Hamiltonian is given by

$$
H=J_{\|} \sum_{i, l=1,2} \overrightarrow{S_{i, l}} \cdot \overrightarrow{S_{i, l}}+J_{\perp} \sum_{i} \overrightarrow{S_{i, 1}} \cdot \overrightarrow{S_{i, 2}}-h \sum_{i, l=1,2} S_{i, l}^{z}
$$

where $l=1,2$ denote the two legs of the ladder, and $h$ the applied magnetic field.

The case when the rung coupling $J_{\perp}$ is much smaller than the interaction $J_{\|}$along the ladder has been studied by a variety of techniques both in thepsence ot 3,11 or in the presence of a magnetic field 19.22 We concentrate here on the opposite limit $J_{\perp} \gg J_{\|}$. In that case the ladder can be mapped onto a single spin 1/2 chain 20,12,21, and we recall the mapping here for completeness. Indeed an individual rung may be in a singlet or a triplet state. Applying a magnetic field brings one component of the triplet closer to the singlet ground state such that for a strong enough magnetic field we have a situation when singlet and $m=-1$ component of triplet create a new effective spin-1/2. It is thus possible if $J_{\perp} \gg J_{\|}$to retain only these two states for all the magnetic field range between $h_{c 1}$ when the gap is broken to $h_{c 2}$ when the ladder is completely magnetized.

One can easily project the original Heisenberg Hamiltonian (11) on the new singlet-triplet subspace

$$
\begin{aligned}
|\tilde{\downarrow}\rangle & =\frac{1}{\sqrt{2}}[|\uparrow \downarrow\rangle-|\downarrow \uparrow\rangle] \\
|\tilde{\uparrow}\rangle & =|\uparrow \uparrow\rangle
\end{aligned}
$$

This leads to the definition of the effective spin $1 / 2$ operators

$$
\begin{aligned}
& S_{1,2}^{+}=\mp \frac{1}{\sqrt{2}} \tilde{S}^{+} \\
& S_{1,2}^{z}=\frac{1}{4}\left[I+2 \tilde{S}^{z}\right]
\end{aligned}
$$

When expressed in term of the effective spin operators (3), the original Hamiltonian (1) becomes

$$
\begin{aligned}
H_{\mathrm{eff}}= & J_{\|} \sum_{i}\left[\tilde{S}_{i}^{x} \tilde{S}_{i+1}^{x}+\tilde{S}_{i}^{y} \tilde{S}_{i+1}^{y}+\frac{1}{2} \tilde{S}_{i}^{z} \tilde{S}_{i+1}^{z}\right] \\
& -\tilde{h} \sum_{i} \tilde{S}_{i}^{z}+C
\end{aligned}
$$

where $C=\left(-\frac{J_{\perp}}{4}+\frac{J_{\|}}{8}-\frac{h}{2}\right) L$ is a simple energy shift and the system is in an effective magnetic field

$$
\tilde{h}=h-J_{\perp}-\frac{J_{\|}}{2}
$$

The Hamiltonian (5) describes a single spin $1 / 2$ chain with a fixed XY anisotropy of $1 / 2$ in an effective magnetic field. In the following we denote with a tilde, the magnetic field $\tilde{h}$, and the magnetization $\tilde{m}$ of the effective spin $1 / 2$ chain. The gaped phase $h<h_{c 1}$ for the ladder corresponds to the negatively saturated magnetized phase for the effective spin chain, whereas the massless phase for the ladder corresponds to the finite magnetization phase for the effective spin $1 / 2$ chain 18 . The field $h_{c 2}$ where the ladder is totally magnetized correspond to the fully magnetized phase for the effective spin $1 / 2$ chain. It is easy to check that

$$
\tilde{h}_{c 1, c 2}=\mp \frac{3 J_{\|}}{2}
$$

\section{SINGLE LADDER}

Before taking into account interladder interactions let us first recall some important consequences of such a mapping for the single ladder. In the process we give a more quantitative calculation for the correlation functions as a function of the magnetic field. The results of this section will be used to study the interladder coupling. We focus here on the massless phase $h_{c 1}<h<$ $h_{c 2}$. To conveniently derive the low energy properties of the effective spin $1 / 2$ chain we use the by now well known bosonization technique. We refer the reader to Ref. 27 29,19 for details and just recall here the main steps.

We first use the Jordan-Wigner transformation 2931 which essentially maps the spin problem onto a problem of interacting fermions on a lattice. For the spin $1 / 2$ system considered here, the corresponding fermionic problem has Fermi momentum $k_{F}=\frac{\pi}{2}$ if $\tilde{h}=0$. Finite magnetic field corresponds to a chemical potential for the fermions. We then perform a linearization around the 
free Fermi points given by $\pm k_{F}$, to obtain an effective low energy continuum fermionic theory and then express the fermion operators in term of bosonic ones related to the fermion density fluctuations using the standard dictionary of Abelian bosonization.

$$
\begin{aligned}
S^{+}(x) & =\frac{e^{-\imath \theta(x)}}{\sqrt{2 \pi a}}\left[e^{-\imath \frac{\pi x}{a}}+\cos 2 \phi(x)\right] \\
S_{z}(x) & =-\frac{1}{\pi} \partial_{x} \phi+e^{\imath \frac{\pi x}{a}} \frac{\cos 2 \phi(x)}{\pi a}
\end{aligned}
$$

Where $S^{+}(x)=\frac{S_{n}^{+}}{\sqrt{a}}, S^{z}(x)=\frac{S_{n}^{z}}{a}$ for $x=n a, a$ being the distance between two nearest neighbors sites along the chain. From now on we take the lattice spacing $a=1$ and measure all distance in units of $a$. The phase $\phi$ is related to the average density of fermions (or equivalently to the uniform spin density along $z$ ) by $S_{z}(x)=-\frac{1}{\pi} \partial_{x} \phi$, whereas $\theta$ is connected to the conjugate momentum $\Pi$ of $\phi\left(\right.$ such that $\left.\left[\phi(x), \Pi\left(x^{\prime}\right)\right]=i \delta\left(x-x^{\prime}\right)\right)$ by $\theta(x)=\int_{-\infty}^{x} d y \Pi(y)$. In a very crude sense $\phi, \theta$ can be viewed as the polar angles of a spin. The low energy properties of the Hamiltonian (5) can be totally described in terms of the boson Hamiltonian

$$
H=\int \frac{d x}{2 \pi}\left[u K(\pi \Pi)^{2}+\frac{u}{K}\left(\partial_{x} \phi\right)^{2}\right]
$$

where $\phi$ has been shifted to absorb the finite magnetization

$$
\phi \rightarrow \phi+2 \tilde{m} x
$$

The only two parameters controlling the low energy properties are the "spin wave" velocity $u$ and a number $K$ called the Luttinger liquid exponent. Both are known exactly for the spin $1 / 2$ chain 32 . For $\tilde{h}=0$ analytic expressions are known

$$
\begin{aligned}
J_{z} / J_{x, y} & =-\cos \pi \beta^{2} \\
1 / K & =2 \beta^{2} \\
u & =\frac{1}{1-\beta^{2}} \sin \left(\pi\left(1-\beta^{2}\right)\right) \frac{J_{\|}}{2}
\end{aligned}
$$

Thus $K=1 / 2$ for an isotropic Heisenberg chain with $\tilde{h}=0$ whereas $K=1$ for the pure XY one. For the Hamiltonian (5) this leads to

$$
K=3 / 4 \quad, \quad u=\frac{3 \sqrt{3}}{2} \frac{J_{\|}}{2}
$$

At finite magnetic field $K$ and $u$ can be obtained by integration of the Bethe ansatz equations and are shown on Figure 2 for the specific case of the XY anisotropy $1 / 2$.
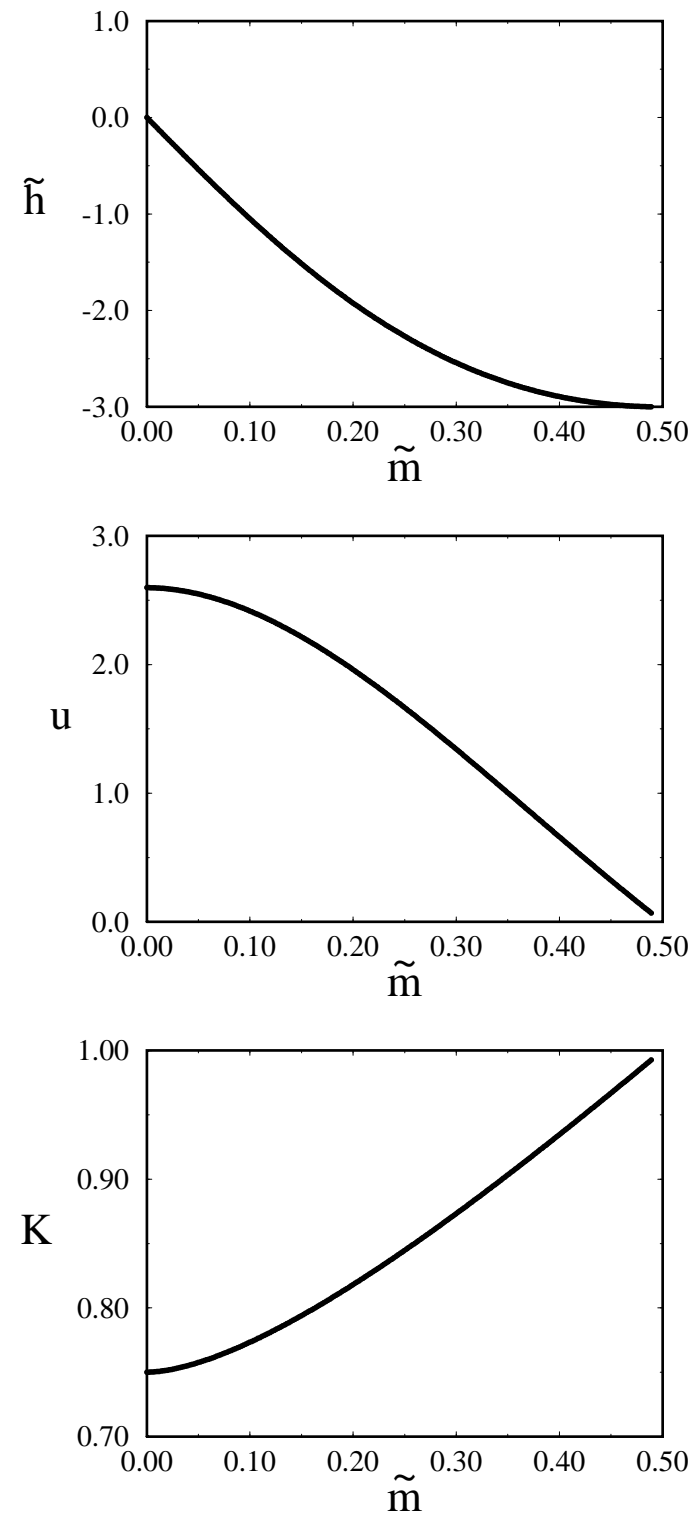

FIG. 2. Magnetic field $\tilde{h}$ and Luttinger liquid parameters $u$ and $K$ for an XY anisotropy of $1 / 2$ plotted as a function of the magnetization $\tilde{m}$ (only positive values are shown, the parameters being symmetric with $\tilde{m} \rightarrow-\tilde{m}) . \tilde{m}=1 / 2$ is the saturated chain. $K=3 / 4$ for zero magnetic field, whereas $K \rightarrow 1$ and $u \rightarrow 0$ close to saturation since the excitations above the ground state become very diluted. $u$ and $\tilde{h}$ are in units of $J_{\|} / 2$.

Close to $h_{c 1}$ or $h_{c 2}$ the number of excitations compared to the fully polarized ground state becomes very small (in the fermionic language one is close to an empty or a full band), and thus $K$ take the value for noninteracting particles $K \rightarrow 1$ regardless of the strength of the original interaction $J_{z} / J_{x y}$.

Since the free boson theory given by (9) is trivially solvable, it is straightforward to calculate the asymptotic decay of the dynamic correlation functions, which are just the ones of a spin $1 / 2$ chain. Using (8), one gets for $T=0$ 3 (for more details see e.g. Ref. 19) 


$$
\begin{aligned}
\left\langle\tilde{S}^{z}(x, \tau) \tilde{S}^{z}(0,0)\right\rangle & =\tilde{m}^{2}+C_{1} \frac{1}{r^{2}}+C_{2} \cos (\pi(1-2 \tilde{m}) x)\left(\frac{1}{r}\right)^{2 K} \\
\left\langle\tilde{S}^{+}(x, t) \tilde{S}^{-}(0,0)\right\rangle & =C_{3} \cos (2 \pi \tilde{m} x)\left(\frac{1}{r}\right)^{2 K+1 /(2 K)}+C_{4} \cos (\pi x)\left(\frac{1}{r}\right)^{1 /(2 K)}
\end{aligned}
$$

where $r=\sqrt{x^{2}+(u \tau)^{2}}$ and $C_{i}$ are constants on which we focus later in this section. When expressed in term of the true magnetization $2 m=1+2 \tilde{m}$ and the original spin operators of the ladder using (3) this gives (e.g. for rung 1)

$$
\begin{aligned}
\left\langle S_{1}^{z}(x, t) S_{1}^{z}(0,0)\right\rangle & =\frac{m^{2}}{4}+\frac{1}{r^{2}}+\cos (2 \pi m x)\left(\frac{1}{r}\right)^{2 K} \\
\left\langle S_{1}^{+}(x, t) S_{1}^{-}(0,0)\right\rangle & =\cos (\pi(1-2 m) x)\left(\frac{1}{r}\right)^{2 K+1 /(2 K)}+\cos (\pi x)\left(\frac{1}{r}\right)^{1 /(2 K)}
\end{aligned}
$$

(where we have dropped the constants $C$ for simplicity). Equ. (14) presents some remarkable features. First, as already pointed out in Ref. 19, low energy modes appear only close to $q \sim 0$ in the $S^{z}$ correlation function or close to $q \sim \pi$ for the transverse one. The $q \sim \pi$ (for $S^{z}$ ) or $q \sim 0$ (for $S^{ \pm}$) mode remain massive. This is in marked contrast to what would happen for a gaped (e.g. dimerized or frustrated) single chain system (in weak coupling) where both the $q \sim 0$ and $q \sim \pi$ would become massless when $h \geq h_{c 1}$. A summary of the massless and massive modes is shown on Figure 3

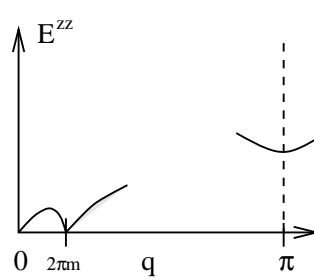

(a)

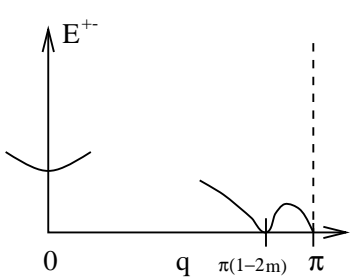

(b)
FIG. 3. Schematic picture of the field dependent dispersion as seen by the $S^{z} S^{z}$ (a) and $S^{+} S^{-}$(b) correlations for fields close to $h_{c 1}$. Only the dominant singularities are shown. In marked contrast with a single chain there is no massless excitations close to $q \sim \pi$ (resp. $q \sim 0$ ) due to the presence of an anisymmetric massive mode, unaffected by the magnetic field 19 .

Such prediction for the ladder correlations should be testable in neutron or resonance experiments. Close to $h_{c 1}\left(\right.$ or $\left.h_{c 2}\right) K \rightarrow 1$ and one recovers the universal exponent for the decay of the correlation functions predicted in Ref. 19. The weak coupling approximation only allowed for a qualitative calculation of the exponents far from $h_{c 1}$ and $h_{c 2}$. For the strong coupling case one can get the full magnetic field dependence as shown in Figure 2. As shown in Appendix A, the whole asymptotic structure of the correlation function is independent of the strength of the coupling $J_{\perp}$ vs $J_{\|}$provided of course that the correct Luttinger liquid exponent are used.

Let us now compute quantitatively the correlation functions. We only focus here on the massless modes. The prefactors in the correlation functions of the spin operators in the XXZ mode-have been computed by Lukyanov and Zamolodchikov33 34. Thus for example for the transverse staggered magnetization we have

$$
(-1)^{n} \tilde{S}^{+}(x=n a, \tau)=\left[F_{\beta} / 8\right]^{1 / 2}(a / u)^{\beta^{2} / 2} e^{i \theta(x, \tau)}
$$

where the expression for the prefactor reads 34

$$
\begin{aligned}
F= & \frac{1}{2\left(1-\beta^{2}\right)^{2}}\left[\frac{\Gamma\left(\frac{\beta^{2}}{2-2 \beta^{2}}\right)}{2 \sqrt{\pi} \Gamma\left(\frac{1}{2-2 \beta^{2}}\right)}\right]^{\beta^{2}} \times \\
& \exp \left\{-\int_{0}^{\infty} \frac{d t}{t}\left(\frac{\sinh \left(\beta^{2} t\right)}{\sinh t \cosh \left[\left(1-\beta^{2}\right) t\right]}-\beta^{2} e^{-2 t}\right)\right\}
\end{aligned}
$$

In the vicinity of the value of interest here $\beta^{2}=2 / 3$ an analytic calculation is possible:

$$
\begin{aligned}
F_{\beta} & =\frac{9}{2 \pi^{2 / 3}}\left[\frac{\Gamma(2 / 3)}{\Gamma(1 / 3)}\right]^{2} e^{-\left(\beta^{2}-2 / 3\right)[\gamma+\ln (3 \pi / 16)+\pi / \sqrt{3}]} \\
& \approx F(2 / 3) \exp \left[-2.173\left(\beta^{2}-2 / 3\right)\right]
\end{aligned}
$$

For more general values of $K$ and $m$ the value of $F$ is shown on Figure 1

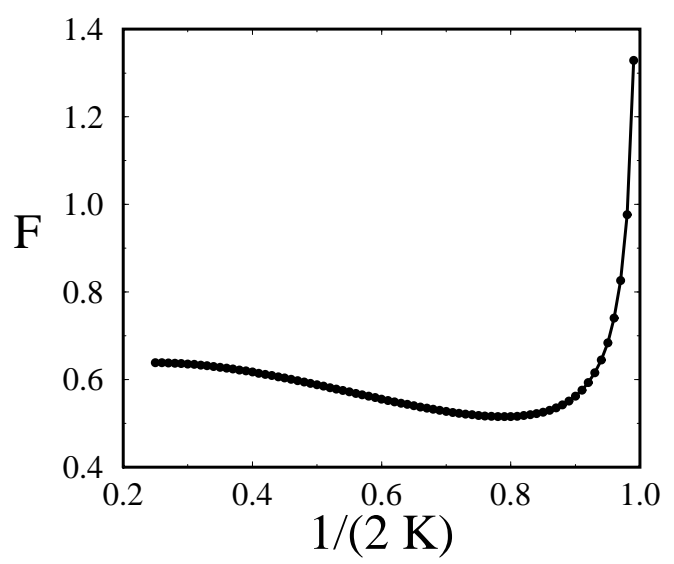




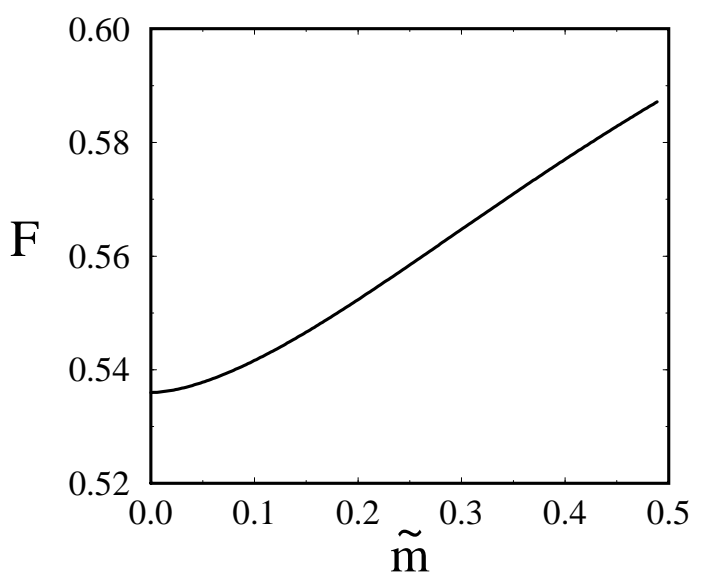

FIG. 4. Prefactor $F$ as a function of the Luttinger parameter $K$. The divergence close to $K=1 / 2$ is due to the appearance of logarithmic cprrections in the correlations functions for the isotropic case 35 . For the specific case of the chain with XY anisotropy $1 / 2$, the prefactor is shown as a function of the magnetization $m$. Note the relatively weak dependence on the whole interval due to the finite XY anisotropy of the effective spin chain.

Using (15) one gets for the staggered part of the transverse spin-spin correlation function of the physical ladder spins as a function of space, time and temperature

$$
=\frac{1}{T^{1 / 3}}\left(\frac{u}{a}\right)^{1 / 3} \frac{3 \sqrt{3} \Gamma(2 / 3)}{16 \sqrt{2} \pi} \approx 0.1 \frac{1}{T^{1 / 3}}\left(\frac{v}{a}\right)^{1 / 3}
$$

Close to $h_{c 1}$ or $h_{c 2}, K \overrightarrow{1}$ (thus $\beta^{2} \rightarrow 1 / 2$ ) and (20) gives back the universal 9 divergence of the relaxation time

$$
\frac{1}{T_{1}} \propto \frac{1}{T^{1 / 2}}
$$

Away from the critical field the exponent increases weakly to $-1 / 3$ at $\tilde{h}=0$. (see also Ref 21,23). The full magnetic field dependence can be obtained from Figure 2 (using $\beta^{2}=1 / 2 K$ and (20)).

Although, as shown in Appendix A, the correlation functions for the strong and weak coupling ladder are smoothly connected, a very interesting question is how the Luttinger liquid parameter varies with the field when going from weak to strong coupling. This is not trivial since for the weak coupling ladder when $\Delta \ll h \ll J_{\|}$ one recovers essentially the Luttinger liquid exponent of a single chain 19 . For an isotropic system this is $K=1 / 2$, i.e. the same value than the universal one close to $h_{c 1}$. If there is $\mathrm{XY}$ anisotropy the parameter $K$ increases and thus $K \geq 1 / 2$. On the other hand for the strongly coupled ladder regardless of the XY anisotropy (provided $J_{z}>0$ ) the luttinger liquid parameter decreases with the field (see (5.11) and Figure 2), giving the very different field dependence shown in Figure 5 .

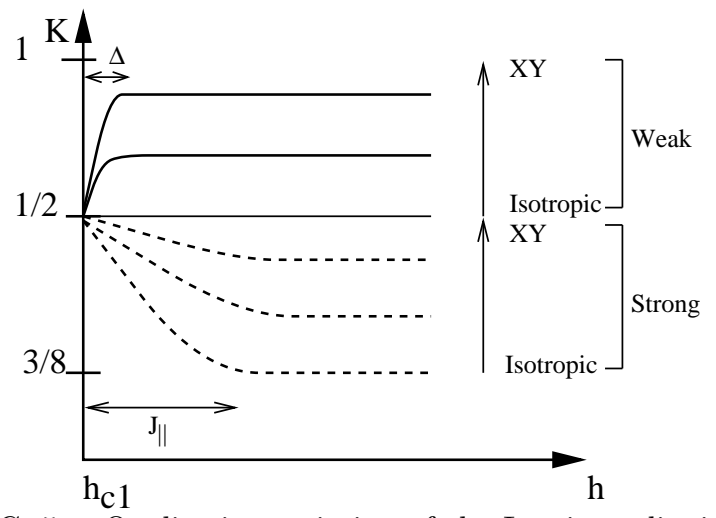

FIG. 5. Qualitative variation of the Luttinger liquid parameter $K$ as a function of the magnetic field both for the weak and strong coupling ladder. Full line is the weak coupling ladder $J_{\perp} \ll J_{\|}$, and dashed line for the strong coupling one $J_{\perp} \gg J_{\|}$. Different curves correspond to different $\mathrm{XY}$ anisotropy from the isotropic case to the XY limit. $\Delta$ is the gap for the weak coupling case. See also Figure for the exact result for the strong coupling ladder (due to the difference of definition (see text) between $K_{\text {weak }}$ and $K_{\text {strong }}$ we plot $K_{\text {strong }} / 2$ ).

It would be extremely interesting to have more quantitative estimates for the behavior shown in Figure 5 . An amusing possibility would be to have, for a certain strength of coupling and a given anisotropy a luttinger liquid parameter totally field independent. 


\section{COUPLED LADDERS}

In order to describe realistic compounds we now take into account an interladder interaction of the form shown in Figure 11 and given by the Hamiltonian

$$
\begin{array}{r}
H_{3 D}=\sum_{\alpha} H_{\text {ladder }}^{\alpha}+J^{\prime} \sum_{\langle\alpha, \beta\rangle} \sum_{i} S_{i, \alpha, 1} \cdot \overrightarrow{S_{i, \beta, 2}} \quad(24) \quad \begin{array}{l}
\text { problem. The coupled ladd } \\
\text { problem of spin } 1 / 2 \text { chains con }
\end{array} \\
H_{\text {coupling }}=-\frac{J^{\prime}}{4} \sum_{\langle\alpha, \beta\rangle}\left[\tilde{S}_{\alpha}^{+} \tilde{S}_{\beta}^{-}+\text {h.c. }\right]+\frac{J^{\prime}}{4} \sum_{\langle\alpha, \beta\rangle} \tilde{S}_{\alpha}^{z} \tilde{S}_{\beta}^{z}+\frac{J^{\prime} z}{8} \sum_{\alpha} \tilde{S}_{\alpha}^{z}
\end{array}
$$

where $\langle\alpha, \beta\rangle$ denotes pairs of nearest neighbors ladders. It is easy to see from Figure 1 that a spin on leg 1 of one ladder can only interact with the spin on leg 2 of the neighboring ladder and vice versa.

Since the interladder coupling is very weak it is again legitimate to map the problem to an effective spin $1 / 2$ problem. The coupled ladder system thus reduces to a problem of spin $1 / 2$ chains coupled by the interaction where $z$ is the coordination number. Because a spin on leg 1 can only be coupled to a spin on leg 2 by $J^{\prime}$ this leads to a ferromagnetic coupling for the XY part of the interchain coupling although the original interladder coupling is antiferromagnetic. There is also a trivial redefinition of the effective magnetic field by the interladder coupling. Although the problem of coupled ladders now look identical to the one of three dimensionally coupled spin 1/2 chain, the physics will be quite different from the standard case 36 of isotropic coupled spin $1 / 2$ chains. Indeed, as we will see below, the XY anisotropy of the effective spin $1 / 2$ chain inherent in the ladder problem, plays a crucial role. The treatment of (25) depends crucially on what is the characteristic energy scale of the interladder coupling when compared to what happens for a single ladder.

\section{A. High density limit}

If one is far enough from $h_{c 1}$ and $h_{c 2}$, the interladder coupling will be small compared to the characteristic energies (Fermi energy for the associated spinless fermion problem) of the single chain. It is then possible to treat the interladder coupling in the mean field approximation while keeping the full single ladder physics. Since the single chain correlation functions along $z$ decays faster than the one in the XY plane (see e.g. (14), three dimensional order will occur first in the XY plane. It is thus possible to neglect in 25 the interchain $\tilde{S}^{z} \tilde{S}^{z}$ coupling, and to retain only the XY part. Note that in that case it is not important whether the interchain coupling is ferro or antiferro since one can go from one to the other by making the gauge transformation $\tilde{S}^{x} \rightarrow-\tilde{S}^{x}$, $\tilde{S}^{y} \rightarrow-\tilde{S}^{y}, \tilde{S}^{z} \rightarrow \tilde{S}^{z}$ on alternating chains. The coupling is just a spin flip term for the spin which in a bosonic representation for the chain is just a hoping term for the bosons (see below). Another way of viewing it is as a
Josephson coupling between the phases $\theta$ of the spins on different chains. This shows that the transition is a normal-superfluid type transition or alternatively is a Bose-condensation transition for the hard core bosons associated with the effective spins. In a pictorial level this says that the orientation of the spins of the ladder in the XY plane want to lock in the same direction as shown in Figure 6. Since we are in $d=3$ (i.e. above the critical dimension for the quantum transition 37 ) the exponents are the mean-field ones $(\nu=1 / 2$ and $\zeta=1)$.

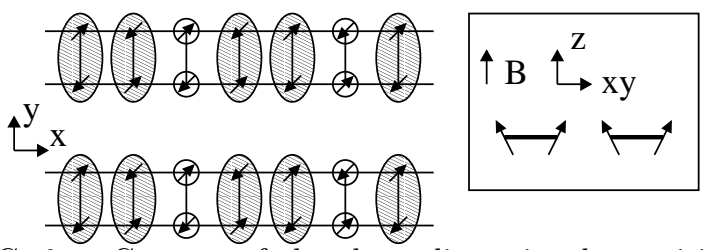

FIG. 6. Cartoon of the three dimensional transition in ladder systems. The direction of the spins in the XY plane tends to lock together between different chains leading to a planar antiferromagnet. Note that the triplet states are in fact delocalized on the ladder and that in the XY plane the spins remains modulated at $q=\pi$ in the ladder direction. We have represented the singlets as shaded boxes. This transition is in the universality class of normal-superfluid transition or Bose-condensation of hard core bosons.

To compute the transition one can use a standard mean-field approach. The transition temperature is given by

$$
\frac{1}{J^{\prime}}=\chi_{\perp}(q=0, \omega=0, T)
$$

where $\chi_{\perp}$ is the single chain transverse staggered susceptibility (wavevector $q$ is to be counted relative to $\pi)$. For the futtinger liquid the susceptibility can be computed 3839

$$
\begin{aligned}
\chi_{\perp}(\omega, q)= & -\frac{F_{\beta}}{8} \frac{a}{v}\left[\sin \left(\frac{\pi \beta^{2}}{2}\right)\left(\frac{2 \pi a T}{u}\right)^{-2+\beta^{2}} B\left(\frac{\beta^{2}}{4}-i s_{+}, 1-\frac{\beta^{2}}{2}\right) B\left(\frac{\beta^{2}}{4}-i s_{-}, 1-\frac{\beta^{2}}{2}\right)\right. \\
& \left.-\frac{\pi}{1-\beta^{2} / 2}\right]
\end{aligned}
$$


where

$$
s_{ \pm}=\frac{\omega-v q}{4 \pi T}
$$

and $B(x, y)=\Gamma(x) \Gamma(y) / \Gamma(x+y)$.

Solving (26) with (27) gives the critical temperature

$$
T_{c}=u\left(\frac{J^{\prime} D_{\beta}}{16 \pi v}\right)^{1 /\left(2-\beta^{2}\right)}
$$

with

$$
D_{\beta}=F_{\beta} \sin \left(\pi \beta^{2} / 2\right)\left[\frac{\Gamma\left(\beta^{2} / 4\right) \Gamma\left(1-\beta^{2} / 2\right)}{\Gamma\left(1-\beta^{2} / 4\right)}\right]^{2}
$$

Given the fact that we used quantitative estimates for the one-dimensional correlation functions and not just asymptotic estimates (29) should even be able to give semi-quantitatively the $T_{c}$ if $J^{\prime}$ can be determined by an independent method. Much more important however, is the field dependence of the $T_{c}$. Indeed since the exponents depend on the field in a non trivial way one can expect a non trivial magnetic field dependence of the three dimensional transition temperature. Close to $\tilde{h}=0$ an analytical solution can be obtained. We have

$$
\frac{d \ln T_{c} / J^{\prime}}{d H}=-\frac{3}{4} \frac{d \beta^{2}}{d H}\left[1.16+\ln \left(v / T_{c} a\right)\right]+\frac{1}{4} \frac{d \ln v}{d H}
$$

It is clear that at small enough $J^{\prime}$ this expression becomes positive and thus magnetic field increases the transition temperature. A numerical estimate of $T_{c}$ is given in Figure 7 .

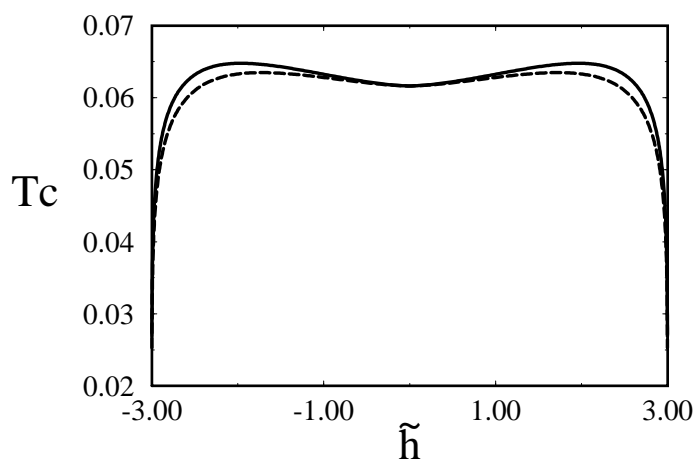

FIG. 7. Three dimensional transition temperature $T_{c}$ as a function of the applied magnetic field $\tilde{h}$ (i.e. for $h_{c 1}<h<h_{c 2}$ ). The full line is the complete solution whereas for the dotted curve the prefactor $F_{\beta}$ has been fixed to its zero field value. Note the minimum at $\tilde{h}=0$

The camel-type structure, instead of the naively expected dromedary one, comes from the competition between the decrease of the exponent when one moves closer to $h_{c 1}$ or $h_{c 2}$ (which leads to an increase of the $T_{c}$ ), with the fact that the excitation velocity $u$ decreases at the same time.

We can also obtain the local susceptibility close to $T_{c}$. In the mean field approximation it is given by

$$
\chi_{l o c}(\omega)=\frac{1}{(2 \pi)^{2}} \int \frac{d q_{1} q_{2}}{\left[\chi_{0}^{-1}-2 J_{1}^{\prime}-2 J_{2}^{\prime}\right]+4 J_{1}^{\prime} \sin ^{2} q_{1} / 2+4 J_{2}^{\prime} \sin ^{2} q_{2} / 2}
$$

When $\tau \equiv \chi_{0}^{-1}(0,0)-2 J_{1}^{\prime}-2 J_{2}^{\prime} \ll J^{\prime}$ one can linearize the sinuses and calculate the integral

$$
\Im m \chi_{l o c}(\omega)=\frac{1}{4 \pi \sqrt{J_{1}^{\prime} J_{2}^{\prime}}} \tan ^{-1}\left[\frac{\Im m \chi_{0}^{-1}}{\Re e \chi_{0}^{-1}-2 J_{1}^{\prime}-2 J_{2}^{\prime}}\right]
$$

In the limit of zero frequency we get

$$
\lim _{\omega \rightarrow 0} \frac{\chi_{l o c}^{\prime \prime}(\omega)}{\omega}=\frac{1}{\pi u} \int \frac{d x\left[\psi\left(1-\beta^{2} / 4+i x\right)-\psi\left(\beta^{2} / 4+i x\right)\right]}{1-\left(T_{c} / T\right)^{2-\beta^{2}}\left|\frac{\Gamma\left(\beta^{2} / 4-i x\right) \Gamma\left(1-\beta^{2} / 4\right)}{\Gamma\left(1-\beta^{2} / 4-i x\right) \Gamma\left(\beta^{2} / 4\right)}\right|^{2}}
$$

Close to $T_{c}$ (34) reduces to a mean field divergence $1 /\left(T-T_{c}\right)^{1 / 2}$. This leads to a similar divergence in the relaxation rate $1 / T_{1}$.

\section{B. Low density limit}

When the magnetic field is close to $h_{c 1}$ or $h_{c 2}$ the above mean field approach on the single chain Luttinger liquid cannot be used. Indeed the energy of the interchain cou- pling becomes larger than the intra chain energy scale, and interchain coupling should be treated from the start. Fortunately the problem is still solvable since the number of excitations above the fully polarized ground state become very small. Let us focus on $h \sim h_{c 1}$, the solution for $h \sim h_{c 2}$ can be deduced by symmetry.

Another useful way of viewing this problem, specially useful when we deal with the low density limit, is given by using the boson representation of spins, instead of the standard Jordan-Wigner fermionic one (the fermions 
have to carry a string). The spins can be represented by hard core bosons. The presence of a boson denotes a spin up state or in the original ladder a triplet on the rung. The hard core constraint ensures that one has only two states (full or empty) on each site to get a faithful representation of the spin $1 / 2$. The problem thus reduces to a problem of hard core bosons with in chain interactions (due to the $\tilde{S}^{z} \tilde{S}^{z}$ term). The interchain coupling Hamiltonian is thus just the kinetic energy interchain hopping term of these bosons

$$
\frac{J^{\prime}}{4} \sum_{i,\langle\alpha, \beta\rangle} b_{i, \alpha}^{\dagger} b_{i, \beta}
$$

Since the bosons are very diluted it is essentially exact to neglect the interactions between them but for the hard core constraint, as indicated by the fact that the Luttinger liquid parameter for a single chain goes to $K=1$ close to $h_{c 1}$. One has thus to solve the problem of a three dimensional gas of hard core bosons with the simple kinetic energy

$$
\begin{aligned}
H_{\text {boson }} & =\frac{J_{\|}}{2} \sum_{i, \alpha}\left(b_{i, \alpha}^{\dagger} b_{i+1, \alpha}+\text { h.c. }\right) \\
& +\frac{J_{\perp}}{2} \sum_{i,\langle\alpha, \beta\rangle}\left(b_{i, \alpha}^{\dagger} b_{i, \beta}+\text { h.c. }\right)
\end{aligned}
$$

To go back to the standard negative hopping one must perform along the chain the gauge transformation

$$
c_{i} \rightarrow(-1)^{i} c_{i}
$$

Since the density is low $(36)$ can be reduced to the continuum limit of bosons with the kinetic energy

$$
E\left(k, k_{\perp}\right)=\frac{k_{\|}^{2}}{2 m}+\frac{k^{2}}{2 M}
$$

The ordering transition thus reduces to the well known problem of the Bose condensation transition for the diluted boson gas 40 . The three dimensional ordered phase corresponds to the superfluid one, whereas the high temperature phase is the normal fluid of bosons. Most physical quantities relevant for the ladder problem can immediately be borrowed from the vast knowledge existing for the diluted boson gas. We will not dwell on all quantities that can be computed but simply give here a few examples.

The critical temperature is known in the limit of low density and is given by the equation 40

$$
\frac{\Lambda}{t_{0}}=\frac{\tilde{h}}{t_{0}}-\frac{2}{(4 \pi)^{3 / 2}} \zeta(3 / 2) T^{3 / 2}=0
$$

where $t_{0}$ is a simple number (scattering matrix for an infinite hard core potential). This leads to a critical temperature varying as

$$
T_{c} \propto\left(h-h_{c 1}\right)^{2 / 3}
$$

and gives back immediately the mean field critical exponents. Of interest is also the total density $\rho$, i.e. the magnetization of the ladder. It is given by

$$
\begin{array}{ll}
\rho=\frac{\tilde{h}}{t_{0}}-\frac{1}{(4 \pi)^{3 / 2}} \zeta(3 / 2) T^{3 / 2} & , \quad T<T_{c} \\
\rho=\frac{1}{(4 \pi)^{3 / 2}} \zeta(3 / 2) T^{3 / 2} & T>T_{c}
\end{array}
$$

Thus two effects occurs. First the magnetization is non monotonous in temperature and increases by a factor of two between $T=T_{c}$ and $T=0$. Second, at criticality $h=h_{c 1}$ the magnetization grows as $T^{3 / 2}$ at very low temperatures (which can be readily seen by computing $\int d^{d} q n_{B}(\epsilon(q))$. This is a different temperature dependence than for independent ladders. In that case it would be given by the excitations of the one-dimensional theory, which are fermionic in nature and have a one dimensional density of state, leading to

$$
m \propto \int_{0}^{\epsilon_{\max }} d \epsilon N_{1 d}(\epsilon) n_{F}(\epsilon) \propto T^{1 / 2}
$$

Looking at the temperature dependence (for very low temperatures) of the the magnetization at criticality should thus provide information on the interladder coupling. Of course at higher temperatures one always recovers the independent ladders behavior (43).

Another important quantity is the NMR relaxation rate. The $\left\langle S^{+} S^{-}\right\rangle$correlation function is here simply given by the bosonic single particle Green's function

$$
\left\langle S^{+}(r, \tau) S^{-}(0,0)\right\rangle=(-1)^{r}\left\langle c(r, \tau) c^{\dagger}(0,0)\right\rangle
$$

The $(-1)^{r}$ factor coming from the transformation (37) implies that the low energy (massless) part of the spectrum which for the spins is around $q \sim \pi$ (see Figure 3)) is given by the small $\omega$ small $q$ Green's function for the bosons. The single particle Green's function can also be computed in the limit of low density 40 and is given in the condensed low temperature phase by

$$
G\left(\omega_{n}, q\right)=\frac{i \omega_{n}+k^{2}+\Lambda}{\omega_{n}^{2}+k^{4}+2 \Lambda k^{2}}
$$

Using the standard formula for the NMR relaxation rate (19) and (45) one obtains

$$
\frac{1}{T_{1}} \propto \frac{T}{\sqrt{\Lambda}}
$$

where $\Lambda$ is given by (39) giving thus a relaxation rate proportional to $T$ at low temperature and diverging close to $T_{c}$ as $1 /\left(T_{c}-T\right)^{1 / 2}$.

The above results could apply to the three dimensional phase of $\mathrm{Cu}_{2}\left(\mathrm{C}_{5} \mathrm{H}_{12} \mathrm{~N}_{2}\right)_{2} \mathrm{Cl}_{4}$, which is a strong coupling ladder 18 where this theory is directly applicable. Whether or not the transition experimentally observed 
is due to the mechanism presented here is still an open question other mechanisms of instability have been proposed2625. Various experiments can be performed to elucidate this point. First since the 3D transition described here is simply an ordering in the direction of the spins in the XY plane its impact on the global global magnetization is very weak, as seems to be the case experimentally. Note however that it does change the temperature dependence of the magnetization at criticality and below $T_{c}$. Other interesting experiments could be a fit of the $h-T_{c}$ relation (40) close to $h_{c 1}$ and more generally the camel-like shape of the phase diagram. Local probes like NMR or neutrons should be perfectly suited to study this transition. NMR could provide a way to map the phase boundary (by looking at the divergence of $\left.1 / T_{1} \sim 1 /\left|T_{c}-T\right|^{1 / 2}\right)$. The $1 / T_{1} \sim T$ law in the low temperature phase could also provide conclusive evidence. Finally since we know that the transition is in the normal-superfluid transition universality class, one could also try to compare the thermodynamic singularities.

\section{CONCLUSION}

We have examined in this work the properties of ladders under magnetic field, and focussed on the gapless phase occuring between $h_{c 1}<h<h_{c 2}$. For a single ladder we computed quantitatively the correlation functions as a functions of the magnetic field. The correlation functions in the ladders have an identical structure both for the weak coupling ladder $J_{\perp} \ll J_{\|}$and the strong coupling one. As in weak coupling an interesting feature in the spin correlation function of the ladder compared to a single chain is the appearance for $h \sim h_{c 1}$ of a low energy mode only close to $q \sim 0$ for the $\left\langle S_{z} S_{z}\right\rangle$ correlation function and close to $q \sim \pi$ for the $\left\langle S_{+} S_{-}\right\rangle$one. A single chain would have had both $q \sim 0$ and $q \sim \pi$ mode at low energy. This prediction should be testable in neutrons experiments. Close to the lower critical field $h_{c 1}$ where the gap is destroyed we recovered the universal exponent 19 for the divergence of the NMR relaxation rate $1 / T_{1} \sim 1 / T^{1 / 2}$.

These results served as a basis to analyze the nature of the phase transition in a system of three dimensionally coupled ladders. This problem falls into the category of Bose condensation of hard core bosons, which allows to obtain many properties of the phase diagram and the ordered phase. The variation of $T_{c}$ with the field has a local minimum at $h=\left(h_{c 1}+h_{c 2}\right) / 2$ leading to an unusual camel-like shape for the phase diagram. Close to $h_{c 1}$ and $h_{c 2}$ the transition is similar to the one of a diluted Bose gas with $T_{c} \sim\left(h-h_{c 1}\right)^{2 / 3}$. The temperature dependence of the $S_{s}$ magnetization goes at criticality $h=h_{c 1}$ from a $T^{1 / 2}$ behavior for independent ladders to a $T^{3 / 2}$ (when interladder coupling intervenes) at low temperatures. The NMR rate diverges close to the transition as $1 / T_{1} \sim\left|T-T_{c}\right|^{-1 / 2}$ and behaves as $1 / T_{1} \sim T$ at low temperatures. These quite distinct features could be used to check whether this transition is the one occuring in the experimental system $\mathrm{Cu}_{2}\left(\mathrm{C}_{5} \mathrm{H}_{12} \mathrm{~N}_{2}\right)_{2} \mathrm{Cl}_{4}$.

\section{ACKNOWLEDGMENTS}

We thank L. P. Levy for many inspiring discussions. A. T. is grateful to University of Paris-Sud, where part of this work was completed, for support and hospitality. T.G. thanks the I.T.P. (Santa Barbara), where the final stage of this work was completed, for support and hospitality. This research was supported in part by the National Science Foundation under grant PHY94-07194.

\section{APPENDIX A: WEAK VS STRONG COUPLING LADDER}

We show in this appendix the connection between the correlation functions for the weak coupling ladder and the strong coupling one. The use of the simple luttinger liquid expressions due to Haldane 41 allows for a more transparent derivation than the one given in Ref. 22 .

For weak $J_{\perp}$ one introduces two boson fields (one for each leg) and it is more convenient to use the symmetric and antisymmetric combinations.

$$
\phi_{1,2}=\frac{\phi_{s} \pm \phi_{a}}{\sqrt{2}}
$$

With the usual representations of the spin operators in terms of the $\phi$ and $\theta$. Since the magnetic field couples only to the symmetric field, it can only remove this gap and the antisymmetric field remains massive even above $h_{c 1}$. To write the correlation functions for the spins in terms of the bosonic fields one use the standard representation of spin in terms of fermion operators and then the expression of this fermions in terms of the bosonic ones for a Luttinger liquid. Let us start with the $S_{z}$ operator, connected with the density of associated fermions.

We use the decomposition of the density or fermion operator in a Luttigner Liquid.

$$
\rho=\rho_{0}-\frac{1}{\pi} \nabla_{x} \phi+\sum_{n} e^{i n(Q x+\phi)}
$$

which contains all harmonics $2 k_{F}, 4 k_{F}$ etc. of the fermion density. Traditionally one only retains the lowest (most singular) harmonic, which leads to the standard expression (8). However here, since here the $2 k_{F}$ component is massive due to the presence of the antisymmetric mode, it makes it necessary to retain the next harmonic. This leads to the spin operators (e.g. for spins on chain 1)

$$
S_{z}=\nabla \phi_{s}+e^{\left.i Q x+\sqrt{2}\left(\phi_{s}+\phi_{a}\right)\right)}+e^{i 2 Q x+2 \sqrt{2}\left(\phi_{s}+\phi_{a}\right)}+\cdots
$$


Since the field $\phi_{a}$ remains massive even above $h_{c 1}$, all correlation functions containing it decay exponentially, and can be neglected at large distance (or time). Thus no $Q$ component appears in the correlation function for the ladder 19 , in marked contrast to the frustrated or dimerized single chain. On the other hand the $2 Q$ term contains $2 \sqrt{2} \phi_{a}$. Although this term is superficially massive, it can be combined with a $\cos \left(2 \sqrt{2} \phi_{a}\right)$ term existing in the Hamiltonian for the ladder, giving rise to the operator

$$
C e^{i\left(2 Q x+2 \sqrt{2}\left(\phi_{s}\right)\right)}
$$

where $c$ is a mere constant. This operator containing only the symmetric field is massless. The long wavelength decay of the correlation function in the weak coupling ladder is thus given by

$$
\left\langle S_{z}(r) S_{z}(0)\right\rangle=\frac{1}{r^{2}}+C^{2} \cos (2 Q)\left(\frac{1}{r}\right)^{4 K}
$$

$$
\left\langle S^{+}(r) S^{-}(0)\right\rangle=(-1)^{r}\left(\frac{1}{r}\right)^{1 / 4 K}+\cos (\pi(1+2 m) r)\left(\frac{1}{r}\right)^{1 / 4 K+4 K}
$$

Thus the expression (14) for the strongly coupled ladder is again similar to this one.

Thus weak and strong coupling ladders are smoothly connected. The crucial reason is that the gap in the antisymmetric degrees of freedom which exists already in the weak coupling ladder is equivalent to the neglect of the two excited states of the triplet performed for the strong coupling. In a system without such an antisymmetric gap (such as a dimerized chain) this smooth continuity would not hold and the weak and strong coupling correlation functions would be radically different.

${ }^{1}$ E. Dagotto and T. M. Rice, Science 271, 618 (1996), and references therein.

${ }^{2}$ F. D. M. Haldane, Phys. Rev. Lett. 50, 1153 (1983).

${ }^{3}$ H. J. Schulz, Phys. Rev. B 34, 6372 (1986).

${ }^{4}$ S. P. Strong and A. J. Millis, Phys. Rev. Lett. 69, 2419 (1992).

${ }^{5}$ S. Gopalan, T. M. Rice, and M. Sigrist, Phys. Rev. B 49, 8901 (1994).

${ }^{6}$ D. G. Shelton, A. A. Nersesyan, and A. M. Tsvelik, Phys. Rev. B 53, 8521 (1996).

${ }^{7}$ S. R. White, R. M. Noack, and D. J. Scalapino, Phys. Rev. Lett. 73, 886 (1994).

${ }^{8}$ A. Sandvik, E. Dagotto, and D. J. Scalapino, Phys. Rev. B 53, 2934 (1996).

${ }^{9}$ K. Hida, J. Phys. Soc. Jpn. 60, 1347 (1991).

${ }^{10}$ D. Poilblanc, H. Tsunetsugu, and T. M. Rice, Phys. Rev. B 50, 6511 (1994).
For the weak coupling ladder since $K \geq 1 / 2$ the $\cos (2 Q)$ term is subdominant and can be safely dropped. To make the connection with the case of strong coupling, where one can have $K<1 / 2$, it must be kept22. Since $Q=\pi(1-2 m)$ it is easy to see that (A5) has exactly the same form than the expression derived for the strongly coupled ladder (14), showing that the two limits are smoothly connected.

Similar results can be obtained for the higher harmonics $2 n Q$. For the transverse magnetization correlation one get in a similar way for the weak coupling ladder

$$
S^{+}=e^{i \theta}\left[(-1)^{i}+\cos (2 \phi)+(-1)^{i} \cos (4 \phi)\right]
$$

where the $\cos (4 \phi)$ term comes again from the higher harmonics. As for the $S_{z}$ component, the $\cos (2 \phi)$ remains massive due to the gap in the $\phi_{a}$ field, whereas the $\cos (4 \phi)$ can again be combined with terms in the Hamiltonian to give a massless term. The final result is

${ }^{11}$ M. Greven, R. J. Birgeneau, and U. J. Wiese, Phys. Rev. Lett. 77, 1865 (1996).

${ }^{12}$ M. Takano et al., Phys. Rev. Lett. 73, 3463 (1994).

13 B. Chiari, O. Piovesana, T. Tarantelli, and P. F. Zanazzi, Inorg. Chem. 29, 1172 (1990).

${ }^{14}$ G. Chaboussant et al., Phys. Rev. B 55, 3046 (1997).

${ }^{15}$ P. R. Hammar and D. H. Reich and C. Brohholm and F. Trouw, cond-mat 9708053 .

${ }^{16}$ S. A. Carter et al., Phys. Rev. Lett. 77, 1378 (1996).

${ }^{17}$ G. Chaboussant and al., Phys. Rev. Lett. 80, 2713 (1998).

${ }^{18}$ G. Chaboussant and al., preprint, to be published in Euro. Phys. Journal B (1998).

${ }^{19}$ R. Chitra and T. Giamarchi, Phys. Rev. B 55, 5816 (1997).

${ }^{20}$ K. Totsuka, Phys. Rev. B 57, 3454 (1998).

${ }^{21}$ F. Mila, preprint, cond-mat/9805029.

${ }^{22}$ A. Furusaki and S. C. Zhang, preprint, cond-mat/9807375.

${ }^{23}$ M. Usami and S. Suga, preprint, cond-mat/9809088.

${ }^{24}$ P. H. Hammar, D. H. Reich, C. Broholm, and F. Trouw, Phys. Rev. B 57, 7846 (1998).

${ }^{25}$ R. Calemczuk and al., preprint, cond-mat/9805237.

${ }^{26}$ N. Nagaosa and S. Murakami, preprint, cond-mat/9802085.

${ }^{27}$ V. J. Emery, in Highly Conducting One-Dimensional Solids, edited by J. T. Devreese and al. (Plenum, New York, 1979), p. 327.

28 J. Sólyom, Adv. Phys. 28, 209 (1979).

${ }^{29}$ I. Affleck, in Fields, Strings and Critical Phenomena, edited by E. Brezin and J. Zinn-Justin (Elsevier Science Publishers, Amsterdam, 1988).

${ }^{30}$ P. Jordan and E. Wigner, Z. Phys. 47, 631 (1928).

${ }^{31}$ M. P. M. den Nijs, Phys. Rev. B 23, 6111 (1981).

${ }^{32}$ F. D. M. Haldane, Phys. Rev. Lett. 45, 1358 (1980).

33 S. Lukyanov and A. B. Zamolodchikov, Nucl. Phys. B 493, 571 (1997).

34 S. Lukyanov, preprint, cond-mat/9712314.

35 T. Giamarchi and H. J. Schulz, Phys. Rev. B 39, 4620 
(1989).

${ }^{36}$ H. J. Schulz, Phys. Rev. Lett. 77, 2790 (1998).

${ }^{37}$ M. P. A. Fisher, P. B. Weichman, G. Grinstein, and D. S. Fisher, Phys. Rev. B 40, 546 (1989).

${ }^{38}$ M. C. Cross and D. S. Fisher, Phys. Rev. B 19, 402 (1979).
${ }^{39}$ H. J. Schulz and C. Bourbonnais, Phys. Rev. B 27, 5856 (1983).

${ }^{40}$ V. N. Popov, Functional Integrals and collective excitations (Cambridge University Press, Cambridge, 1987).

${ }^{41}$ F. D. M. Haldane, Phys. Rev. Lett. 47, 1840 (1981). 